\title{
CRISPR/Cas9 Mediated Genome Editing in Crop Plants ${ }^{\#}$
}

\author{
Abdul Razaque Memon ${ }^{1, a, *}$ \\ ${ }^{1}$ Department of Molecular Biology and Genetics, Faculty of Science and Letters, Uşak University, 64000 Uşak, Turkey
}

${ }^{*}$ Corresponding author

\begin{tabular}{|c|}
\hline$\Delta$ \\
\hline $\begin{array}{l}{ }^{\#} \text { This study was presented as an online } \\
\text { presentation at the } 2^{\text {nd }} \text { International } \\
\text { Journal of Agriculture - Food Science } \\
\text { and Technology (TURJAF 2021) } \\
\text { Gazimağusa/Cyprus Research Article }\end{array}$ \\
\hline Review Article \\
\hline
\end{tabular}

Received : 04/11/2021

Accepted : 22/12/2021

\author{
A B S T R A C T
}

Recently, most genomic research has focused on genome editing methods to develop new technologies that could be easy, reliable, and feasible to edit plant genomes for highly productive agriculture. Genome editing is based on alternating a specific target DNA sequence by adding, replacing, and removing DNA bases. This newest technology called CRISPR/Cas9 seems to be less time-consuming, more effective and used in many research areas of plant genetic research. CRISPR/Cas9 systems have many advantages in comparison with ZFNs and TALENs and has been extensively used for genome editing to many crop plant species. Around 20 crop species are successfully worked out for trait improvements, for example, yield improvement, disease resistance, herbicide tolerance, and biotic and abiotic stress management. This review paper will overview recent advances in CRISPR/Cas genome editing research in detail. The main focus will be on the use of CRISPR/Cas9 technology in plant genome research.

Keywords:

CRISPR

Cas9

Genome editing

Plants

ZFNs

Türk Tarım - Gıda Bilim ve Teknoloji Dergisi, 9(sp): 2396-2400, 2021

\section{Tarla Bitkilerinde CRISPR/Cas9 Yöntemi ile Genom Düzenleme}

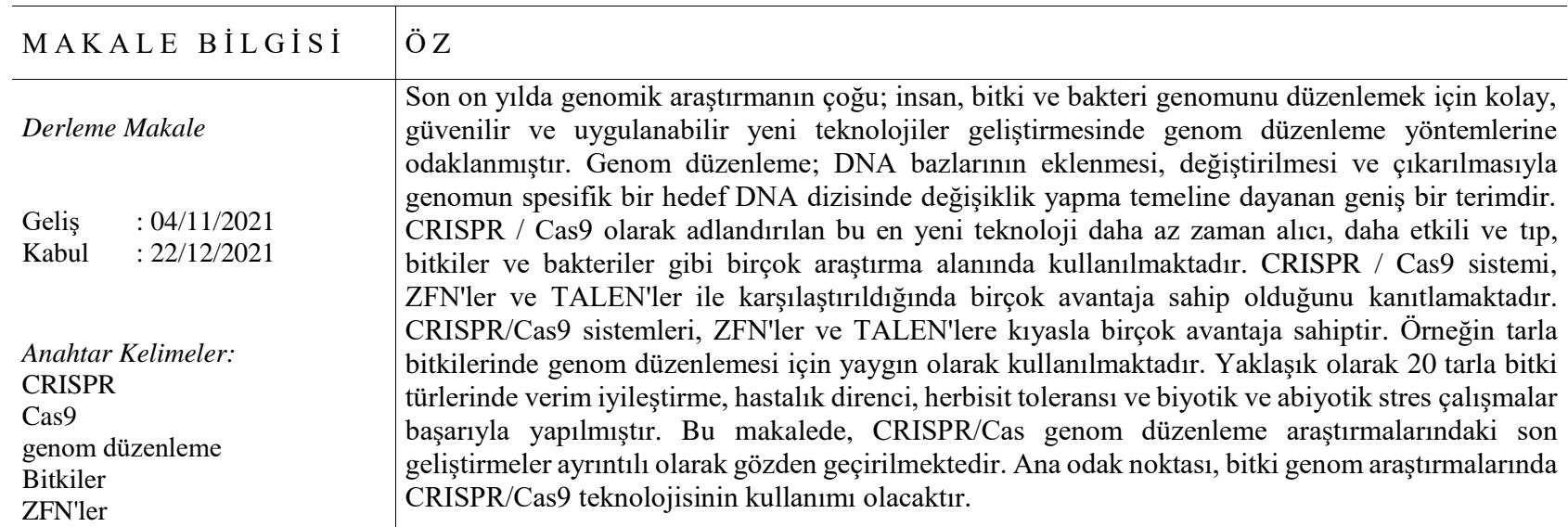




\section{Introduction}

The genetic variations in plants was earlier achieved through random mutagenesis methods such as EMS mutagenesis and $\gamma$-radiation. However, despite apparent success with this approach, a need for a more precise methodology arose. Thus, research has led to ways to precisely manipulate specific genomic sequences. Recently, targeted plant genome editing has emerged as the preferred method for plant engineering, slowly but surely replacing classical plant breeding and transgenic approaches. In order to create specific, desirable mutants of a targeted gene, double-strand breaks (DSB) are introduced in the DNA. These DSBs will trigger one of the independent endogenous DNA repair pathways: nonhomologous end joining or homologous recombination, both resulting in chromosomal changes. These changes can lead to a weakened gene mutation or a loss-of-function gene mutation (Sonoda et al., 2006). Several genetic tools have been developed in recent years that can generate targeted DSBs (Gaj et al., 2013). These tools use site-specific nucleases to introduce targeted DSBs in DNA. The most prominent and successful tools are the zinc-finger nucleases (ZFN's) and the transcription activator-like effector nucleases (TALEN's). ZFN's and TALEN's are artificial bipartite enzymes that consist of a modular DNA binding domain that is programmable and the FokI nuclease cleavage domain that interacts to form chimeric proteins. ZFN's and TALEN's have been used extensively in site-specific genome editing with great success (Wood et al., 2011; Jankele R., Svoboda P., 2014). The TALENs were seen as a revolution in the field of sitespecific nucleases and were considered one of the ten significant breakthroughs during the last decade (Alberts., 2012). Despite the significant contribution, the application of this method has revealed several drawbacks. These drawbacks include very high technological demands, the requirement of highly sophisticated designs, and demanding assembly and delivery. All these requirements are the reason TALEN's have not found wide usage in plant genome editing. A wide variety of species, including many important agricultural plants, have been left widely ignored due to the sheer difficulty of applying TALEN's. However, a new method is recently developed to utilize site-specific nucleases and promises to cover most of TALEN's and ZFN's drawbacks.

This new genome editing system is called the CRISPR/Cas9 system (CRISPR being an acronym for clustered regularly interspaced short palindromic repeats, and Cas 9 being an acronym for CRISPR associated protein 9). The CRISPR/Cas9 system was derived from the adaptive immune system of Streptococcus pyogenes (Jinek et al., 2012, Wright et al., 2016). The components of the CRISPR/Cas9 system are given in Figure 1.

The double-stranded DNA stands for a specific genomic locus. The irregular shape in orange refers to the Cas9 endonuclease, which opens up the DNA double stands for cleavage in the diagram. The single-strand sequence is single guide RNA (sgRNA), but only the RNA sequence in red (called a spacer, part of the crRNA) specifies the Cas9 endonuclease involved in cut or a double-strand break (DSB) in the genome. The target DNA sequences are shown in black, which pairs with the spacer sequence (in red). The required protospacer adjacent motif
(PAM) NGG ( $\mathrm{N}$ refers to one of the four nucleotides A, T, $\mathrm{G}$, and $\mathrm{C}$ ) next to the targeted genomic site is shown in green (adapted from http://www.systembio.com/cas9).

In order to utilize the CRISPR/Cas9 system, researchers first had to customize the initial system found in bacteria and archaea. They reprogrammed the initial system using customizable small non-coding RNAs (Jinek $M$ et al., 2012). This customization resulted in the initial three-component system, consisting of Cas9, crRNA (CRISPR RNA), and tracrRNA (transactivating crRNA), reduced into a two-component system. The crRNA and tracrRNA components were joined together into a singleguide RNA (sgRNA) that can guide the Cas9 to a predetermined site in the genome (Jiang and Doudna 2017, Chen et al. 2019, Anzalone et al. 2020).

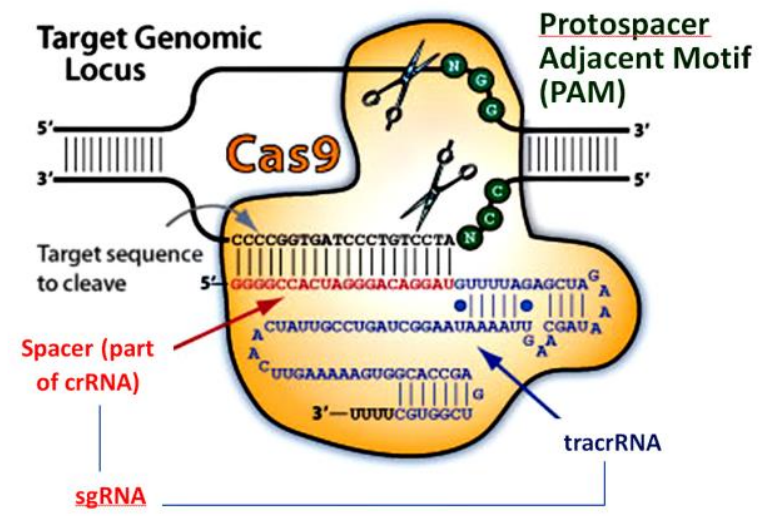

Figure 1. A diagram for the RNA-guide endonuclease system CRISPR/Cas9

\section{Cas9 nuclease}

Cas9 is a large multidomain, and multifunctional DNA nuclease (1,368-amino acid- S. pyogenes Cas9) guided to a DNA target sequence adjacent to the adjacent protospacer motif (PAM) by sgRNA (a complex of two non-coding RNA's: the crRNA and tracrRNA) (Anders et al. 2014, Jiang and Doudna 2017, Anzalone et al. 2020). The crystal structures of Cas9 endonucleases of different sub-types revealed a conserved core and a bi-lobed architecture with adjacent active sites and two nucleic acid binding grooves. The two lobes include a large globular recognition (REC) lobe connected to a small nuclease (NUC) lobe. The REC lobe is a Cas9-specific functional domain composed of REC1 and REC2, and a long a-helical arginine-rich domain referred to as the Bridge Helix (Jiang and Doudna 2017). The NUC lobe accommodates two nuclease domains, RuvC and $\mathrm{HNH}$, and a PAM-interacting domain (PI domain). Two nucleic acid binding grooves, a wide major groove, and a narrow minor groove, are located within the REC and NUC lobes, respectively (Figures 1 and 3 ). Cas9 is a flexible protein that operates alone to bind and cleave the DNA target sequence-dependent. Singleparticle electron microscopy revealed that Cas9 is maintained in an auto-inhibited conformation in the absence of nucleic acid ligands and switches to an active form upon guide RNA loading (Jinek et al. 2014, Jiang and Doudna 2017). 
For the application of the CRISPR/Cas9 technology, a wide array of different optimized versions of codons of Cas9 were used for different plant. In summary, all versions tested were working, some with higher efficiency than others (Figure 2).

\section{Single-guide RNAs (sgRNAs)}

SgRNAs used in the CRISPR/Cas9 system are chimeric RNAs, created by fusing tracrRNA and crRNA (Chen et al. 2019, Anzalone et al. 2020). SgRNAs are designed to match a 20 bp target sequence in the DNA followed by an adjacent protospacer motif (PAM) sequence of NGG. This NGG is required for cleavage, although it does not appear in the sgRNA. The exact bases of the sgRNA and location are very variable since they depend on the promoter used and the target sequence DNA (Bandyopadhyay et al. 2020).

The sgRNA guide sequence is located on the 5 ' end of the sgRNA and is the part of the sgRNA that confers target specificity. This means that modification of this guide sequence is the primary tool to create siRNA with various target specificity. The guide sequence has a canonical length of $20 \mathrm{bp}$. However, recent studies suggest that this length can vary without compromising target specificity. SgRNA with a deviation of 2 to $3 \mathrm{bp}$ in length did not suffer from errors in the target recognition, but the extent to which this deviation is tolerable is unknown. It is reported that mismatches of a few bp did not interfere with normal function; however, a study revealed that these mismatches should be kept under 4 bp (Fu et al., 2013). The Cas9 protein is then guided to specific genomic sites by the sgRNAs that recognize the NGG-type protospacer adjacent motif and targets DNA sequences through Watson-Crick base pairing (Figure 3).

Cas9 protein (light blue) is guided by a structure formed by a sgRNA, in green (composed of a crRNA containing 20nt segment which determine the target specificity, and a transactivating tracr RNA which stabilizes the structure and activates Cas9 to cleave the target DNA-protospacer). PAM sequence directly downstream from the target DNA (protospacer-adjacent motif, in yellow) is required for DNA cleavage by Cas9 (see Figure 3A). This DSB causes random mutations when repaired by the error-prone NHEJ pathway or precise gene modification repaired by the error-free HDR pathway (see Figure 3B). Gene is modified in four different ways, which are as follows: (a) Repair by NHEJ usually results in the insertion (green) or deletion (red) of random base pairs, causing gene knockout by disruption. (b) If a donor DNA is available, which is simultaneously cut by the same nuclease leaving compatible overhangs, gene insertion by NHEJ can also be achieved. (c) HR with a donor DNA template can be exploited to modify a gene by introducing precise nucleotide substitutions or (d) to achieve gene insertion Adapted from Bortesi and Fisher 2014) (Figure 3 B).

\section{CRISPR/Cas Technology Using Transient Expression Assays}

Transient expression assays such as protoplast transformation and in planta expression using Agrobacterium tumefaciens transient expression (agroinfiltration) could be used to test the CRISPR-Cas system in plants. Mutations introduced via both NHEJ and HDR) pathways have been reported. Several studies generated the whole plants that carry mutations at the targeted loci (Belhaj et al. 2013, Lozano-Juste and Cutler, 2014). In order to check for the presence of Cas9/sgRNA-induced mutation, the restriction enzyme (RE) site loss method would be the best choice for usage. This method is a type of mutation detection system which could be applied in many sequenced genes. By using this method, the RE-resistant band can be cloned. The exact nature of the mutations is then revealed by sequencing individual clones (Belhaj et al., 2013).

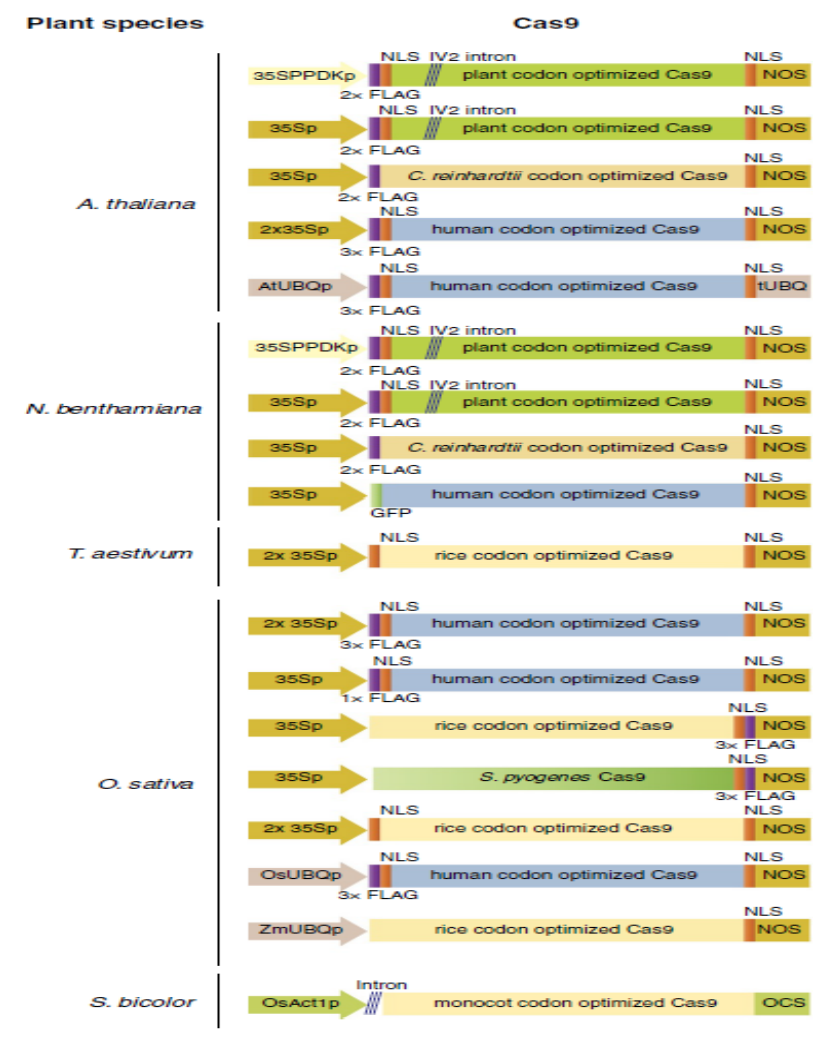

Figure 2. Cas9 variants used for genome editing in plants (Courtesy of Belhaj et al., 2013).

Cas9 was tagged with either FLAG or GFP and expressed in the plant under the control of various constitutive promoters. NLS, nuclearlocalized signal.
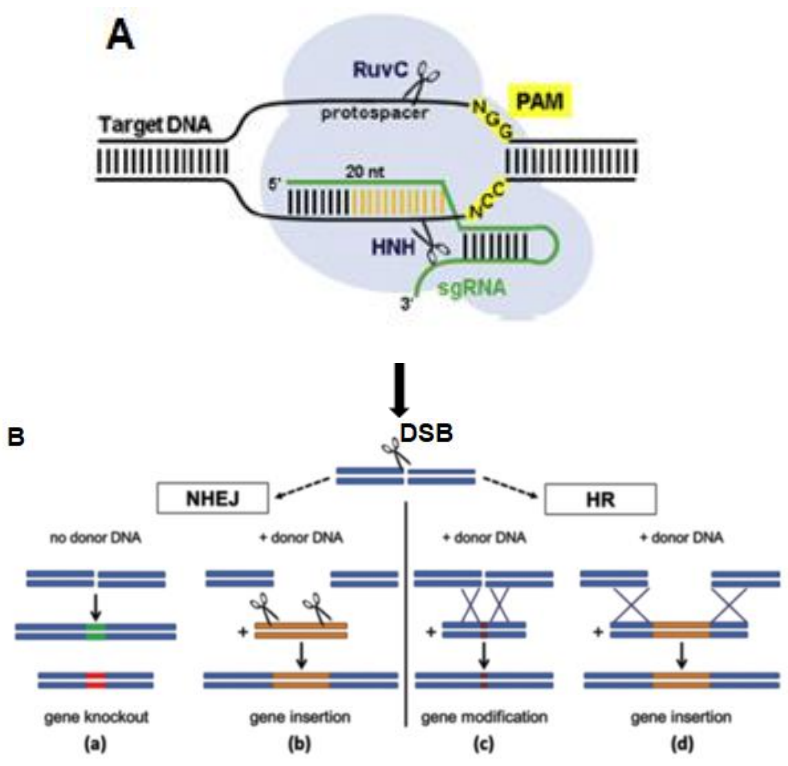

Figure 3. Genome editing in plants with site-specific nucleases 


\section{Advantages of CRISPR-Cas9 Technology over ZFNs and TALENS}

Everything that could be done with CRISPR-Cas9 technology fundamentally could also be achieved to some extent with ZFNs or TALENs. An enormous increase in research on CRISPR-Cas9 technology has proven to be of great importance when studying its advantages. One of such advantages is that CRISPR-Cas9 technology does not require protein engineering steps, and thus it makes it easier to check for target genes. Unlike ZFNs and TALENs, the ease of multiplexing in CRISPR-Cas9 technology has proven to be one of its most significant advantages, which means that several genes could be edited simultaneously with the same technique, the study on genomic deletions and inversions could also be achieved. Multiplex editing with ZFNs and TALENs requires dimeric proteins, which need to be separated, whereas in CRISPR-Cas9 technology, the monomeric Cas9 protein, and some sequence-specific gRNAs are required (Bortesi and Fischer, 2014). In comparison with ZFNs and TALENs, the CRISPR-Cas9 system can differentiate between target DNA or RNA.

\section{Target specificity of the CRISPR-Cas9 in plants}

The ability to make specific changes at targeted genomic sites in complex organisms is crucial in many research areas, especially in plant research. There are many successful applications where CRISPR-Cas9 technology could be used because of its high efficiency and relatively low cost. However, this technology has been of the biggest concern because it can cause off-target mutagenesis. Target specificity is an essential issue in all genome editing methods. Several factors could affect Cas9/sgRNA targeting specificity, which could be classified in following categories: 1) intrinsic specificity, which is encoded in the Cas9 protein, which resolves the relative importance of each position in the sgRNA for target recognition, and 2) approximate abundance of effective Cas9/sgRNA complex concerning effective target concentration (Wu et al., 2014). Targeting Cas 9 to a specific region in the genome appears to affect the specificity predominantly, and besides this, the sequence of $\operatorname{sgRNA}$ alone also affects the specificity. Any alterations of the sgRNA sequence may affect Cas9 specificity. Some of them include changes that cause the off-target effect, alternations of concentration of sgRNA, variations of alternations affecting the chromatin environment of the target DNA sequence, etc. (Wu et al.,2014, Azalone et al. 2020).

The CRISPR/Cas9 technology has been applied in model plants (Nicotiana benthamiana, Arabidopsis thaliana) and crops (rice, wheat). The Cas9 nuclease and the sgRNA matching the gene of interest are co-expressed using Agrobacterium tumefaciens as a vector in $N$. benthamiana leaves. Then, the genomic DNA is extracted from the leaf tissues or protoplasts and subjected to PCR amplification. To check for the presence of Cas9/sgRNAinduced mutation, the restriction enzyme (RE) site loss method is used. The RE-resistant band can be cloned by this method. The exact nature of the mutations can be checked by sequencing individual clones (Belhaj et al., 2013).

\section{Applications of CRISPR-Cas9 in Crop Plants}

Food insecurity and low dietary quality are responsible for the impairments of physical and mental development, several infectious diseases, and high numbers of premature deaths (Development Initiatives 2018). Eliminating or reducing these problems and achieving sustainable development goals, zero hunger, and improved nutrition require major global food systems transformations. Classical plant breeding methods have considerably impacted high yield, especially during the last 100 years (Huang et al. 2002; Evenson and Gollin 2003). But despite this, chronic hunger is still widespread in many poor and developing countries, albeit the global proportion of hungry people was reduced from over $50 \%$ in the first half of the twentieth century to around $11 \%$ today (FAO). Nowadays, new plant breeding technologies, including genetically modified and gene-edited crops, are needed for improving sustainable agricultural development and food security while addressing the shortcomings of the Green Revolution. These technologies could contribute to higher crop yields, lower chemical fertilizers, pesticides, better crop resilience to climate stress, reduced postharvest losses, and better nutritious foods.

During the last 20 years, new breeding technologies were developed, for example, genome editing, including CRISPR/cas9, and is now widely used for crop improvement. One of the purposes of the CRISPR-Cas9 technology is the improvement of plant breeding, which could modify multiple traits. Beyond genome editing in plants, CRISPR-Cas9 technology could be used for ectopic regulation of gene expression, revealing the secrets of gene functions. Another application that will be of great concern in the future is the targeted insertion of transgenes in molecular farming to produce specific proteins in plants. The use of a catalytically inactive version of Cas9 known as dead Cas9 (dCas9) has proven to have many vital roles in regulating gene expression (Miki et al., 2021). The ability of dCas9 to fuse either with transcriptional repressor or activator has demonstrated the importance of dCas9 in gene regulation processes. Delivery of specific cargos to targeted genomic regulations is another use of dCas9 (Bortesi and Fischer, 2014; Wu. et al.,2014). CRISPR/Cas9 techniques have been extensively used for genome editing to many crop plant species; around 20 crop species are successfully worked out for trait improvements, for example, yield improvement, disease resistance, herbicide tolerance, and biotic and abiotic stress management (Chen et al. 2019, Bandyopadhyay et al., 2020).

Numerous publications on CRISPR-Cas9 application in tomato and other fruit crops have been reported (Chen et al. 2019, Wang et al. 2019). The research in CRISPR-Cas9 in fruit crops is classified into four groups. 1. Resistance into biotic stresses, 2. Resistance to abiotic stress, 3. Improvement of fruit quality, 4. domestication of some fruit crops.

\section{Concluding Remarks and Outlook}

The simplicity and robustness of CRISPR/Cas9 technology make it an attractive genome editing tool in plant research. The CRISPR/Cas9 system has been rapidly and effectively adapted in both model and crop plants and 
demonstrated a desirable efficacy in site-specific gene targeting. By its many applications, this technique has become one of the most used and reliable techniques recently. Some of the advantages of CRISPR/Cas9 technology include its accessibility, versatility, and cost. Besides genome editing, this technique could be used in many different research areas that cover gene discovery and trait development. Our better understanding of this system would lead to the discovery and design of new genome editing tools and help further improve plant genetic research.

\section{References}

Alberts B. 2012. The Breakthroughs of 2012. Science, 338:1511. Anders C, Niewoehner O, Duerst A, Jinek M. 2014. Structural basis of PAM-dependent target DNA recognition by the Cas 9 endonuclease. Nature 513, 569- 573.

Anzalone AV, Koblan LW, Liu DR. 2020. Genome editing with CRISPR-Cas nucleases, base editors, transposases and prime editors, Nat Biotechnol. 38: 824-844.

Bandyopadhyay A, Kancharla N, Javalkote V, Dasgupta S, Brutnell TP. 2020. CRISPR-Cas12a (Cpf1): A Versatile Tool in the Plant Genome Editing Tool Box for Agricultural Advancement, Frontiers in Plant Science, Volume 11| Article 584151.

Belhaj K, Chaparro-Garcia A, Kamoun S, Nekrasov V. 2013. Plant genome editing made easy: targeted mutagenesis in model and crop plants using the CRISPR/Cas system. Plant Methods. 9:39, 1-10.

Bortesi L, Fischer R. 2014. The CRISPR/Cas9 system for Plant genome editing and beyond. Biotechnology Advances 33. 4152.

Chen K, Wang Y, Zhang R, Zhang H, Gao C. 2019. CRISPR/Cas Genome Editing and Precision Plant Breeding in Agriculture, Annu. Rev. Plant Biol. 70:28.1-28.31.

Development Initiatives. 2018. Global Nutrition Report 2018. Bristol: Development Initiatives. 9.

Evenson RE, Gollin D. 2003. Assessing the Impact of the Green Revolution, 1960-2000. Science 300: 758-762.

Fu Y, Foden JA, Khayter C, Maeder ML, Reyon D, Joung JK, Sander JD. 2013. High frequency off-target mutagenesis induced by CRISPR-Cas nucleases in human cells. Nat Biotechnol.
Gaj T, Gersbach CA, Barbas CF. 2013. ZFN, TALEN, and CRISPR/Cas-based methods for genome engineering. Trends Biotech 31: 397-405.

Huang J, Pray C, Rozelle S. 2002. Enhancing the Crops to Feed the Poor. Nature 418: 678-684.

Jankele R, Svoboda P. 2014. TAL effectors: tools for DNA targeting. Brief Funct Genomics. 13: 409-19.

Jiang F, Doudna JA. 2017. CRISPR-Cas9 structures and mechanisms, Annu. Rev. Biophys. 2017. 46: 505-29.

Jinek M, Jiang F, Taylor DW, Sternberg SH, Kaya E, Ma E, Anders C, Hauer M, Zhou K, Lin S et al. (2014). Structures of Cas9 endonucleases reveal RNA-mediated conformational activation. Science, 343:1247997.

Jinek M, Chylinski K, Fonfara I, Hauer M, Doudna JA, Charpentier E. 2012. A Programmable Dual-RNA-Guided DNA Endonuclease in Adaptive Bacterial Immunity. Science, 337:816-821.

Lozano-Juste J, Cutler SR. 2014. Plant genome engineering in full bloom. Trends in Plant Science. Vol 19. No 5.

Miki D, Zinta G, Zhang W, Peng F, Feng Z, Zhu J-K. 2021. CRISPR/Cas9-Based Genome Editing Toolbox for Arabidopsis thaliana, Sanchez-Serrano, JJ, Salinas, J (Eds), Arabidopsis Protocols, Methods in Molecular Biology, Vol.2200, Springer Nature.

Sonoda E, Hochegger H, Saberi A, Taniguchi Y, Takeda S. 2006. Differential usage of nonhomologous end-joining and homologous recombination in double strand break repair. DNA Repair (Amst) 5:1021-1029.

Wang T, Zhang H, Zhu H. 2019. CRISPR technology is revolutionized the improvement of tomato and other fruit crops. Horticulture Research. 6, 77: 1-13

Wood AJ, Lo TW, Zeitler B, Pickle CS, Ralston EJ, Lee AH, Amora R, Miller JC, Leung E, Meng X, Zhang L, Rebar EJ, Gregory PD, Urnov FD, Meyer BJ. 2011.Targeted genome editing across species using ZFNs and TALENs. Science, 333:307.

Wright AV, Nunez JK, Doudna JA. 2016. Biology and applicationof CRISPR systems: Harnessing nature's tool box for genomic engineering. Cell 164:29-44.

Wu X, Kriz AJ, Sharp PA. 2014. Target specificity of the CRISPR-Cas9 system. Quant Biol. 2: 59-70

Xuebing Wu, Andrea JK, Sharp PA. 2014. Target specificity of CRISPR-Cas9 system, Quantitative Biology. 2(2): 59-70. 